Revue Gouvernance

Governance Review

GOUVERNANCE

GOU SRNANCL

\title{
Le nouveau management public comme prémisse aux transformations des systèmes de santé nationalisés : les cas du Québec et du Royaume-Uni Managerialism and the transformation of nationalized health
systems: the cases of Quebec and the U.K.
}

\section{Mélanie Bourque}

Volume 4, numéro 1, 2007

URI : https://id.erudit.org/iderudit/1039117ar

DOI : https://doi.org/10.7202/1039117ar

Aller au sommaire du numéro

Éditeur(s)

Centre d'études en gouvernance de l'Université d'Ottawa

ISSN

1912-0362 (numérique)

Découvrir la revue

Citer cet article

Bourque, M. (2007). Le nouveau management public comme prémisse aux transformations des systèmes de santé nationalisés : les cas du Québec et du Royaume-Uni. Revue Gouvernance / Governance Review, 4(1).

https://doi.org/10.7202/1039117ar

\section{Résumé de l'article}

Les systèmes de santé font l'objet du débat politique depuis les années 1990. Ces débats, qui portent sur le vieillissement de la population, l'évolution technologique et les demandes accrues des usagers, ont amené la plupart des pays occidentaux à réformer leur système. Malgré les orientations différentes qu'ont pris les réformes, les transformations des systèmes de santé nationalisé, mandaté et entrepreneurial ont principalement visé la réduction des coûts. Cet article tente de montrer que l'application des principes du nouveau management public aux systèmes de santé du Québec et du Royaume-Uni s'est principalement faite dans le but de contourner les contraintes que sont :

l'universalité, le financement public et l'unicité de gestion. Ces caractéristiques fondamentales des systèmes nationalisés ont conduit les décideurs à créer un " marché intérieur " qui vise la mise en concurrence des établissements de santé les uns par rapport aux autres en ayant pour objectif principal l'atteinte des résultats préalablement fixés. L'application du nouveau management public transforme les fondements des systèmes de santé du Québec et du Royaume-Uni, et par le fait même, remet en question leur catégorisation en tant que système nationalisé. 


\title{
Le nouveau management public comme prémisse aux transformations des systèmes de santé nationalisés: Les cas du Québec et du Royaume-Uni ${ }^{1}$
}

par Mélanie Bourque

\section{Résumé}

Les systèmes de santé font l'objet du débat politique depuis les années 1990. Ces débats, qui portent sur le vieillissement de la population, l'évolution technologique et les demandes accrues des usagers, ont amené la plupart des pays occidentaux à réformer leur système. Malgré les orientations différentes qu'ont pris les réformes, les transformations des systèmes de santé nationalisé, mandaté et entrepreneurial ont principalement visé la réduction des coûts. Cet article tente de montrer que l'application des principes du nouveau management public aux systèmes de santé du Québec et du Royaume-Uni s'est principalement faite dans le but de contourner les contraintes que sont : l'universalité, le financement public et l'unicité de gestion. Ces caractéristiques fondamentales des systèmes nationalisés ont conduit les décideurs à créer un «marché intérieur » qui vise la mise en concurrence des établissements de santé les uns par rapport aux autres en ayant pour objectif principal l'atteinte des résultats préalablement fixés. L'application du nouveau management public transforme les fondements des systèmes de santé du Québec et du Royaume-Uni, et par le fait même, remet en question leur catégorisation en tant que système nationalisé.

\section{Managerialism and the transformation of nationalized health systems: the cases of Quebec and the U.K.}

by Mélanie Bourque

\begin{abstract}
Health systems have been central in political debates since the 1990s. These debates focussed on population aging, technological evolution and patients' demands, and drove Western countries to reform their systems. Even if the specific reforms varied from country to country, they included mandated, nationalized and entrepreneurial systems transformation with cost reduction/containment as the principal target. This article shows that Quebec and Great Britain's health systems directed governments to apply new public management principles to circumvent constraints such as universality, public financing and unity of management. These central nationalized system basic characteristics encouraged health authorities to create an "internal market" which aims to put the facilities in competition with each other in a result-based management perspective. The application of new public management changed the bases of Quebec's and Great Britain's health systems, and consequently, has led to the questioning of their definition as nationalized systems.
\end{abstract}

\footnotetext{
${ }^{1}$ Cet article s'inscrit dans le cadre d'une recherche beaucoup plus large sur l'impact des systèmes de santé sur les inégalités sociales de santé.
} 


\section{Introduction}

Au nom du vieillissement de la population, de l'évolution technologique et des demandes accrues des usagers, la plupart des pays occidentaux ont procédé à des réformes de leur système de santé. L'abondante recherche sur l'organisation des systèmes de santé porte, le plus souvent, sur la structure de la prestation des soins. Peu se sont intéressés à analyser les réformes des systèmes de santé dans la perspective plus large de l'analyse des politiques publiques. C'est ce que nous nous proposons de faire ici.

Au moyen de la comparaison entre les systèmes de santé québécois et britannique, nous montrerons que les systèmes nationalisés (Roemer, 1993) imposent davantage de contraintes aux changements. Ces contraintes qui caractérisent ce type de système, dans un contexte de coupures budgétaires, ont conduit les décideurs à effectuer des transformations «de l'intérieur » et d'imposer de façon plus extensive les principes du nouveau management public que dans les autres systèmes de santé comme ceux de la France ou des Pays-Bas. Le but de cet article est, dans un premier temps, de dégager les principes qui ont été imposés dans le cadre des récentes réformes aux systèmes de santé nationalisés du Québec et du Royaume-Uni. Dans un deuxième temps, il vise à expliquer ces transformations, ce qui conduira à établir la manière dont les systèmes universels (nationalisés) de soins de santé ont été réformés, ainsi que les types de contraintes qui se sont imposés dans le cadre de ces changements.

L'article est divisé en trois parties. La première vise à expliquer les prémisses théoriques de notre analyse, la seconde permettra d'exposer les grandes caractéristiques des systèmes de santé du Québec et du Royaume-Uni. Enfin, la troisième aura pour but de montrer les principes qui ont été adoptés dans le cadre des réformes des systèmes de santé québécois et britannique.

\section{Néo-institutionnalisme et systèmes de santé}

Pour comparer les systèmes de santé nationaux, nous prenons comme point de départ la prémisse néo-institutionnaliste qui attribue aux institutions un rôle structurant dans le domaine de la définition des politiques publiques. Nous partons ainsi du fait que chaque système est nécessairement le résultat de choix politiques oumis à des contraintes exogènes, par exemple, les transformations économiques structurelles, et endogènes, comme le parti au pouvoir ou le système de santé déjà en place. L'idée n'est toutefois pas de rendre uniquement compte de la convergence et de la divergence qui existent entre les systèmes de santé. Malgré le fait qu'il s'agisse d'une caractéristique inhérente à la comparaison (Jenson, 2003), s'arrêter-là nous semble peu porteur. Nous cherchons donc à dépasser ce débat entre les chercheurs qui s'intéressent à la protection sociale. S'il est important de bien saisir les éléments qui caractérisent le changement, se limiter à décrire la convergence des politiques à cause de la mondialisation (Mishra, 1999) ou d'identifier les différences comme facteurs inéluctables ne permet pas d'expliquer les facteurs qui ont initié, qui participent et qui découlent des transformations de la protection sociale. $\mathrm{Ce}$ reproche est d'ailleurs souvent adressé à la littérature qui porte sur les États providence puisqu'elle se limite souvent à décrire le changement sans produire d'explications complexes ou théoriques (Pierson, 2000). 
Plusieurs chercheurs ont toutefois développé des concepts qui permettent d'expliquer ces changements et d'identifier leurs causes et leurs conséque nces. L'analyse des politiques publiques dans le domaine de la protection sociale montre, que depuis le début des années 1980, on assiste au passage à un nouveau paradigme (Jenson, 2006). Certains fondent leur explication sur le plan macro et insistent sur une mutation générale du rôle de l'État. Le concept d'État post-moderne proposé par Chevallier (2003) ou celui du Shumpetarian Workfare State définit par Jessop (1997) représentent des exemples types de ce courant analytique. D'autres, même s'ils s'accordent avec ces derniers, se centrent davantage sur l'analyse des politiques publiques en développant, ce l'on appelle des middle range theory (Lecours, 2005). Par exemple, en proposant le concept de l'État LEGO, Denis Saint-Martin et Jane Jenson (2005), ont défini les transformations de la protection sociale. Quant à lui, Peter Hall (1993) s'appuie sur le concept de policy paradigm alors que Ferrera et Hemerjick (2003) proposent celui de de recalibration. Les systèmes de santé nationaux n'échappent pas à ces transformations paradigmatiques (Jenson, 2003).

\section{Le NMP comme nouveau paradigme de la gestion des systèmes de santé nationalisés}

En s'opposant à l'administration publique classique qui était selon ses tenants, trop rigide, réglée et inefficace, le nouveau management public (NMP) vise, dans le but d'accroître l'efficacité de la «gestion publique », la transposition des principes de la gestion qui prévalent dans le secteur privé. Comme nous procéderons à l'analyse de systèmes de santé, il est intéressant de noter que cette transformation de l'administration publique weberienne fondée sur la rationalité est apparue au cours des années 1980, en même temps que la remise en question du rôle de l'État et de la protection sociale (Chevallier, 2003). On peut affirmer que les caractéristiques principales du NMP sont celles de la gestion par résultats, de la livraison de services de qualité, de la reddition de compte centré sur le client, de l'évaluation de programme, de l'imputabilité des fonctionnaires, de la privatisation et de la gestion horizontale des ressources humaines, de la mise en concurrence, de la décentralisation ou du délestage de pouvoir à de nouvelles instances (Rouillard, Monpetit et al., 2004). La plupart de ces principes ont été institués dans les systèmes de santé du Québec et du Royaume-Uni.

On le constatera dans les pages qui suivent, chacun des pays a procédé à des degrés différents à des transformations importantes de leur système de santé en appliquant les principes du nouveau management public. Il s'agira d'abord de proposer une typologie des systèmes de santé qui permet de catégoriser les systèmes de santé et d'en analyser les changements. On procédera ensuite à la présentation des cas du Québec et du RoyaumeUni. Cette démonstration permettra de montrer, comment les systèmes nationalisés ont été transformés depuis les années 1980.

Les systèmes de santé 
L'ensemble des pays occidentaux ont procédé à des réformes de leurs systèmes de santé. Les raisons évoquées par les gouvernements sont multiples. Ils insistent sur la crise des finances publiques, sur l'augmentation des coûts des systèmes de santé liés aux changements démographiques et technologiques ainsi qu'au vieillissement de la population. On met, par ailleurs, l'accent sur la qualité des soins, leur efficacité et leur efficience, ainsi que sur l'équité. On peut sans aucun doute affirmer que, comme dans le cas des autres politiques sociales, les réformes des systèmes de santé s'inscrivent dans une transformation plus large de la protection sociale.

L'adoption de la perspective théorique néo-institutionnaliste a conduit à choisir la grille d'analyse de Roemer qui définit les systèmes de santé comme le résultat de l'histoire, du développement économique et de l'idéologie politique dominante des régimes politiques dans lesquels ils prennent forme. Ainsi, chaque pays définira de manière particulière son système de santé. Par contre, pour des fins analytiques, Roemer propose des idéaux-types qui englobent les différents systèmes de santé que voici : le système nationalisé offre une couverture universelle des soins de santé aux citoyens, de plus, la production des services et le financement du système proviennent majoritairement du public (le Canada, le Royaume-Uni et la Suède); le système mandaté offre une assurance obligatoire qui est généralement financé par l'employeur et l'État, de même, la production et la prestation des services sont publiques ou privées (en France, en Allemagne et aux Pays-Bas); enfin, le système entrepreneurial est constitué d'assurances privée collective ou individuelle et se caractérise par la production de services privés (aux États-Unis et au Mexique). L'utilisation de ces différents idéaux types permet de saisir les fondements des systèmes de santé tout en étant en mesure de dégager les éléments qui témoignent de leurs transformations. Sur un plan plus général, il importe de considérer également des facteurs exogènes qui influencent les systèmes de protection sociale et, par le fait même, les systèmes de santé. Pensons, par exemple, aux crises économiques, à la mondialisation des marchés et aux crises des finances publiques qui exercent une pression sur les États (Chevallier, 2003). Toutefois, comme cet article s'intéresse plus particulièrement à l'organisation des soins, il s'agira d'explorer les éléments suivants : le type d'assurance, le financement, l'organisation des soins, et les indicateurs choisis pour évaluer les systèmes de santé.

\section{Le Québec et le Royaume-Uni}

Les systèmes de santé du Royaume-Uni et du Québec se présentent comme deux systèmes de santé nationalisés. Les deux systèmes d'inspiration beveridgienne ${ }^{2}$ sont fondés sur les principes de l'universalité et de besoin. Leur financement est assuré par le biais de l'impôt sur le revenu et fondé sur l'unicité de la gestion du système.

\footnotetext{
${ }^{2}$ Les systèmes de protection sociale sont généralement classés selon deux catégories : le régime bismarckien présent en Europe et le régime beveridgien duquel s'inspire la majorité des pays anglo-saxons. Le régime bismarckien est fondé sur le principe de la cotisation sociale des travailleurs. Ainsi, chaque travailleurs, cotise aux caisses d'assurance sociale et a ainsi accès aux prestations et services. Au contraire, le régime beverigdgien est fondé sur le principe de l'universalité indépendamment de l'activité professionnelle, c'est par l'impôt sur le revenu que les citoyens cotisent au régime de protection sociale. Ainsi, chaque citoyen, indépendamment de sa situation ou de son activité professionnelle a droit aux prestations et services mis en œuvre.
} 
Contrairement aux systèmes mandatés où les individus sont couverts en fonction de leur activité professionnelle ou même les systèmes entrepreneuriaux qui sont largement privés, le système nationalisé est beaucoup plus complexe à transformer. Ses caractéristiques inhérentes que sont l'universalité, le financement public et particulièrement l'unicité de gestion le rendent plus rigide. Il s'agit d'ailleurs d'une des principales critiques des tenants de la réforme en profondeur d'un tel système (Quesnel Vallée, Bourque et al, 2006), mais là n'est pas notre propos. Cette contrainte au changement a conduit les décideurs à contourner cette soi-disant rigidité pour appliquer ce qu'il est convenu d'appeler les principes du nouveau management public.

\section{Le cas du Québec}

Le système de santé québécois est soumis à la Loi canadienne sur la santé adoptée en 1984. Celle-ci stipule que tous les Canadiens doivent recevoir des soins de santé gratuits en fonction de leur lieu de résidence et de leurs besoins.

La Commission Castonguay-Neveu conduit, en 1972 à l'adoption de la Loi sur les services de santé qui sera remise en question quelque vingt années plus tard par la Commission Rochon (1985). Le gouvernement dirigé alors par le Parti libéral de Robert Bourassa, ne donnera toutefois pas suite aux recommandations de cette commission qui proposait, entre autres, un système centré sur l'atteinte de résultats, une approche populationelle permettant d'agir sur les facteurs déterminants des problèmes ainsi que des mesures d'encouragement au rendement en fonction de résultats obtenus. C'est en 1997, confronté aux compressions budgétaires, que le gouvernement du Parti québécois mettra en œuvre le virage ambulatoire. L'objectif principal visé était clair : il s'agissait de procéder à une désinstitutionnalisation des soins de santé en mettant l'accent sur les services de maintien à domicile des personnes âgées. De plus, on s'engagea dans un programme de mise à pied de nombreux travailleurs du réseau.

En 2001, le gouvernement du Québec toujours dirigé par le Parti québécois mettra sur pied une nouvelle commission sur les soins de santé qui sera, cette fois, présidée par Michel Clair. Le rapport Clair recommande alors au gouvernement d'améliorer les services de première ligne et de faire en sorte que l'ensemble des Québécois ait un médecin de famille; il propose en même temps la création d'un système d'assurance contre la perte d'autonomie et l'ouverture au privé dans la santé. En fait, la réforme préconise un système de santé dans lequel la responsabilité individuelle prend une nouvelle importance (Charbonneau, 2003). Comme dans le cas de la commission Rochon, le gouvernement ne donnera pas de suite immédiate aux recommandations de cette commission.

Parallèlement, le gouvernement fédéral met sur pied la Commission Romanow qui arrive à des conclusions différentes de celle du rapport Clair, puisqu'elle sera moins ouverte à l'implication du privé dans le système de santé. En effet, le rapport qui s'intitule Guidés par nos valeurs : l'avenir des soins de santé au Canada ${ }^{3}$ propose une réforme du système

\footnotetext{
${ }^{3}$ Voir : http://www.hc-sc.gc.ca/francais/soins/romanow/index.html, consulté le 30 janvier 2007.
} 
de santé canadien qui vise également une refonte de son organisation. Toutefois, les conclusions du rapport mettent un accent particulier sur l'accès universel aux soins de santé qui, selon Romanow, représente une valeur canadienne fondamentale.

C'est en 2003 que le gouvernement du Parti libéral procédera à une réforme du système de santé en adoptant la Loi sur les agences de développement de réseaux locaux de services de santé et de services sociaux. La réforme Couillard de 2003, qui s'inscrit dans le plan plus large de la modernisation de l'État du gouvernement Charest, a modifié de façon importante le réseau de la santé du Québec. En cherchant à implanter l'approche populationnelle qui vise à établir des liens entre l'état de santé de la population et les établissements qui rendent les services, le gouvernement a procédé à une refonte du système de santé sans transformer de manière importante les critères des systèmes nationalisés que sont l'universalité, la prestation de soins selon les besoins et le financement par l'impôt. Les transformations ont plutôt eu lieu dans l'organisation du système de santé et ont particulièrement affecté le principe de l'unicité de gestion.

Le premier élément est celui de la décentralisation, avec la création des Agences de santé et de services sociaux dont la mission est de réorganiser le système et de s'assurer, entre autres, du développement de soins en fonction de la population de chaque territoire. Conformément aux principes du nouveau management public, le gouvernement crée de nouvelles instances dans le but de déléguer la gestion du réseau. Le principe de l'unicité de gestion est, dès lors, remis en question. Les agences issues des anciennes Régies de la santé et des services sociaux se voient ainsi confier davantage de pouvoir en matière de gestion. Les agences dressent les portraits de santé de la population qu'elles desservent, elles sont responsables de produire des données sociodémographiques, sociosanitaires, socio-économiques, ainsi que sur l'utilisation des services. Le principal objectif est de définir les caractéristiques populationnelles comme le statut socio-économique, les maladies les plus fréquemment diagnostiquées, les habitudes de vie, etc.

Le second élément consiste en l'intégration du réseau, notamment avec la création des Centres de santé et de services sociaux (CSSS) qui résulte de la fusion entre CLSC, CHSLD et Centre Hospitalier et qui vise la création de zone de soins et des réseaux locaux de santé et de services sociaux. On peut citer à titre d'exemple la création du réseau local du cœur de l'île qui résulte de la fusion entre l'hôpital Jean-Talon, les CLSC Villeray et Petite-patrie ainsi que les Centres d'hébergement Paul-Gouin et Auclair. Cette nouvelle institution est sous l'égide de l'Agence de santé et de service sociaux de Montréal. Malgré la volonté d'implanter l'approche populationnelle, on peut affirmer que derrière ces fusions se cachent, entre autres, la rationalisation de la gestion et la volonté de la réduction des coûts.

Conformément aux principes de l'approche populationnelle, le gouvernement a créé des zones de soins qui desservent une population particulière. De plus, chaque CSSS doit produire un projet clinique dont le but est de faciliter les parcours de soins des patients et ainsi d'améliorer l'efficacité du système. Enfin, on peut avancer qu'une résultante des projets cliniques est de placer les établissements de santé en situation de concurrence les 
uns par rapport aux autres en ayant pour objectif l'atteinte, voire le dépassement, de résultats qui ont été fixés.

L'accent mis sur la participation des usagers dans la définition du système de santé, constitue un autre élément important de cette réforme. Non seulement ceux-ci doivent être représentés sur les conseils d'administration des établissements, mais on les consulte de plus en plus dans l'évaluation de la performance des établissements. À ce titre, la transformation de l'évaluation du système de santé fait ressortir de façon intéressante les transformations qu'a subies le système. En parallèle, l'abolition du Conseil de santé et de bien-être et la création de la fonction de Commissaire à la santé et au bien-être, adopté en 2005, mais appliqué en 2006, constituent certainement un des éléments central de la transformation du réseau. Le Conseil de santé et de bien-être avait pour mission première de fournir des avis au Ministre, son abolition a été effectuée au nom de la transparence. Le Commissaire à la santé et au bien-être a pour mission d'informer et de consulter les citoyens ainsi que d'évaluer l'atteinte de résultats et de recommander des améliorations ${ }^{4}$. Il est toutefois intéressant de constater que le CNBS fondait sa légitimité sur un groupe de 23 membres nommés par le Gouvernement. La mission du Commissaire est, quant à elle, plus large; elle vise, comme nous l'avons mentionné plus haut, à faire une évaluation du système, mais, dans cette perspective, elle s'appuie sur un Forum de citoyens nommé par le Commissaire lui-même. De plus, ce dernier, ne relève pas de l'Assemblée nationale, mais bien du Ministre de la Santé et des Services sociaux. Cette décision prise par le gouvernement Charest implique une transformation importante de l'évaluation qui porte sur la qualité des soins et la santé de la population. On a par ailleurs introduit un volet important d'indicateurs qui vise dorénavant à mesurer la satisfaction des usagers. On mesure plus particulièrement l'état de santé, la qualité des soins, la satisfaction des usagers et la performance. Comme on le verra un peu plus loin, l'évaluation du système de santé du Québec est substantiellement différente de celle qui est effectuée au Royaume-Uni laquelle est davantage axée sur la satisfaction du client. Malgré le fait que l'on s'appuie sur certains indicateurs de satisfaction, l'évaluation demeure tout de même centrée sur l'état de santé de la population.

On peut avancer que la mise en concurrence des établissements de santé que l'on peut associer au benchmarking, ainsi que la décentralisation, ressortent comme des éléments forts de la réforme. Le cas de la Montérégie l'illustre parfaitement, puisque l'Agence a créé un site Internet $^{5}$ qui vise l'aide à la prise de décision et qui permet aux établissements de se fixer des objectifs pour améliorer les services et de se comparer avec les autres établissements. Cette mise en concurrence n'est toutefois pas jusque-là aussi accentuée qu'au Royaume-Uni, où l'on retrouve des éléments similaires à la réforme québécoise.

Avant de poursuivre sur le système de santé du Royaume-Uni, il apparaît important de mentionner l'adoption sous bâillon par le gouvernement Charest du projet de Loi 33 en décembre 2006. Cette nouvelle loi, qui donne suite à l'Arrêt Chaoulli, modifie de façon significative le système de deux façons, à savoir, d'abord, en ouvrant certains secteurs de

\footnotetext{
${ }^{4}$ Voir : http://www.csbe.gouv.qc.ca/site/fr commissaire mission.phtml, consulté le 4 avril 2007.

${ }^{5}$ Voir : http://www.rrsss16.gouv.qc.ca/continuums/index.html, consulté le 4 mars 2007.
} 
soins (les chirurgies de la hanche, du genou et de la cataracte) à l'assurance privée; ensuite, en visant le recours de plus en plus grand aux partenariats publics-privés (PPP). Nous pouvons toutefois avancer que sans l'Arrêt Chaoulli, le gouvernement libéral n'aurait probablement pas procédé à une telle modification du système ${ }^{6}$. Seul l'avenir pourra confirmer si cette brèche va conduire à la redéfinition du système nationalisé.

\section{Le cas du Royaume-Uni}

Le système britannique a subi des réformes importantes depuis le milieu des années 1980 qui ont transformé le système de santé en profondeur. La dernière réforme remet en question la catégorisation du Royaume-Uni comme système de santé nationalisé, il en sera d'ailleurs question plus loin.

L'arrivée au pouvoir du gouvernement Thatcher a, comme on le sait, transformé radicalement l'intervention de l'État dans la plupart des secteurs. La réduction des dépenses qui était le leitmotiv de ce gouvernement a métamorphosé la protection sociale ainsi que le système de santé. Par exemple, en 1984, Thatcher implante le General Management Function, qui visait, entre autres, la réduction du personnel et l'atteinte de résultats. Durant les années 1990, le National Health Service \& Community Care Act crée déjà un "marché intérieur" qui implique que les autorités publiques en matière de santé n'assurent plus la gestion des hôpitaux, mais deviennent acheteurs de services. On a ainsi encouragé la compétition, mais également les différences locales dans la production et la prestation de soins (Rivett, 1998). On peut, encore une fois, affirmer que les principes du NMP sont utilisés pour contourner les contraintes du système, dans ce cas-ci, par la création d'un marché intérieur.

En 1997, dès son arrivée au pouvoir, le gouvernement Blair met en oeuvre une réforme intitulée The New NHS, Modern Dependable ${ }^{7}$. Celle-ci favorise une meilleure communication à l'intérieur des services, met l'accent sur la qualité et préconise une révision de l'organisation du système qui vise une meilleure intégration. Ces modifications seront suivies, en 1998, par d'autres transformations, notamment la création de trusts qui assurent la gestion et la livraison des soins ainsi que la création de la Commission for Health Improvement, qui évalue la performance du système.

En 2000, le gouvernement de Tony Blair procédera à une autre réforme, le NHS Plan, qui mettra l'accent sur l'augmentation du rôle du privé dans le système de santé. Les hôpitaux se voient maintenant gérés par des firmes privées, le but étant évidemment de réduire les coûts du système et d'en améliorer l'efficacité. On fait appel aux partenariats

\footnotetext{
${ }^{6}$ L'Arrêt Chaoulli peut être interprété comme une fenêtre d'opportunité pour l'ouverture à l'assurance privée. Plusieurs juristes dont Marie-Claude Prémont affirment d'ailleurs que pour répondre au jugement, le gouvernement n'était pas obligé de permettre le recours à l'assurance privée. Il s'agit donc d'un choix politique rendu possible grâce à une interprétation d'un jugement de la Cour Suprême. (Voir Marie-Claude Prémont, « L'affaire Chaoulli et le système de santé du Québec. Cherchez l'erreur. Cherchez la raison », 2006, Revue de droit de McGill/McGill Law Journal,Vol. 51, p. 167-195.)

${ }^{7}$ Royaume-Uni. Gouvernement du Royaume-Uni, The New NHS. Modern. Dependable. Crown copyright, 1997.
} 
publics privés (PPP) en confiant à des gestionnaires privés la gérance des établissements de santé. En 2001, on procède à d'autres changements qui accentuent les éléments de la réforme précédente puisque l'on met en place un système de paie lié à la performance en même temps qu'on procède à un alignement de l'évaluation et de la gestion des ressources humaines sur le système privé. Jusque-là, on assiste à une transformation importante qui vise, entre autres, la mise en concurrence des établissements. Ce phénomène sera toutefois accentué de façon importante en 2006 puisque le gouvernement de Blair adoptera une autre réforme qui pousse un peu plus loin encore les principes adoptés lors des réformes précédentes.

La réforme de 2006 est davantage centrée sur le patient-client et s'appuie de manière beaucoup plus marquée sur le principe du benchmarking que le système de santé du Québec. Le gouvernement insiste sur le fait que la réforme du National Health Service vise à développer un système de santé qui met le patient de l'avant et qui est développé en sa fonction ${ }^{8}$. Le but, comme dans le cas québécois est d'améliorer la performance et l'efficacité du système. La prise en compte du patient semble dans les deux cas être la voie à suivre. En effet, depuis janvier 2006, le système de santé du Royaume-Uni s'ouvre à la concurrence en permettant aux patients de choisir leur établissement de santé en fonction d'indicateurs de performance rendus publics ${ }^{9}$.

Les patients sont informés sur plusieurs aspects des établissements dont les services hospitaliers comme le stationnement ou la disponibilité d'un téléphone, la qualité des soins, les firmes de gestionnaires qui les gèrent et l'appréciation des patients qui ont fréquenté cet établissement de santé. Dans la région du sud-ouest, par exemple, l'hôpital de Derriford semble avoir, d'après les indicateurs recueillis sur le site Internet, de piètres résultats : il assurerait une qualité de service correct, mais utiliserait mal ses ressources. De plus, il annulerait régulièrement des opérations, aurait de longues listes d'attente et serait considéré comme moins convivial que les deux autres établissements de la région. La production de ce type d'information est certainement de lourde de conséquences pour un hôpital, mais elle l'est encore davantage dans le cas du Royaume-Uni, puisque le financement est accordé à l'établissement en fonction de sa performance.

Ainsi, non seulement les patients se voient contraints de faire des choix à partir des indicateurs de performance, mais les budgets sont accordés en fonction du nombre de patients qui y sont traités. L'argent des soins suit donc le patient. Comme le mentionne très clairement le gouvernement : «Best provider attracts more patients ${ }^{10}$. Les «meilleurs » se verront atribuer des budgets plus élevés. Cet aspect particulier du financement du système de santé du Royaume-Uni représente très clairement, comme dans le cas du Québec, mais de façon plus accentuée sans doute, une application du principe du benchmarking qui suppose que les services seront améliorés s'ils sont mis en concurrence et systématiquement comparés les uns aux autres.

${ }^{8}$ http://www.dh.gov.uk/PolicyAndGuidance/OrganisationPolicy/HealthReform/fs/en, consulté le 12 décembre 2006.

${ }^{9}$ Voir :http://www.dh.gov.uk/PolicyAndGuidance/OrganisationPolicy/HealthReform/fs/en, consulté le 15 janvier 2007.

${ }^{10}$ Voir, http://www.direct.gov.uk/en/HealthAndWellBeing/index.htm, consulté le 30 janvier 2007. 
L'évaluation des soins est davantage axée sur le traitement des plaintes et la gestion de la performance. La Commission for Healthcare Audit and Inspection (CHAI) est l'institution qui procède à l'évaluation des systèmes de santé. Plusieurs indicateurs sont évalués, mais on met principalement l'accent sur la qualité des soins, l'efficience, le fait que le système soit axé sur le patient, l'équité, la prestation efficace des soins, la satisfaction des usagers et la performance. Contrairement au Québec, on remarque qu'il n'y a pas ou peu d'évaluation de l'état de santé de la population et que les notions d'efficacité et d'efficience du système de santé sont centrales.

\section{Le nouveau management public comme prémisse de l'évolution du système nationalisé?}

Le système nationalisé, qui repose sur les prémisses de l'assurance publique universelle et le financement public ainsi que l'unité de gestion, impose plus de contraintes aux changements. Ces facteurs contraignants ont conduit les décideurs à procéder à des transformations de l'intérieur. En effet, on remarque que jusqu'à maintenant les réformes ont davantage visé l'organisation des soins, plutôt que la remise en question de l'universalité de ces deux systèmes de santé.

On peut affirmer que les réformes du modèle nationalisé optent pour des trajectoires qui sont similaires, puisque dans les deux cas on a procédé à la transformation de l'intérieur de l'organisation des soins de santé. Pour ce faire, les deux systèmes ont adopté les principes du NMP qui s'inspirent de la gestion prévalant dans le secteur privé. Les principales caractéristiques de chacune des réformes le confirment. Le Québec et le Royaume-Uni semblent avoir opté pour la création d'un «marché intérieur » qui met l'accent sur la mise en concurrence des établissements pour réduire les dépenses et accroître l'efficacité du système.

Plusieurs de ces principes peuvent être identifiés dans les réformes des systèmes de santé du Québec et du Royaume-Uni. Les deux systèmes ont eu recours à la décentralisation/déconcentration. Au Québec, on a creé les Agences de santé et de services sociaux, alors qu'au Royaume-Uni on a plutôt attribué la gestion des établissements à des gestionnaires privés. Dans les deux cas, on a recours à la gestion par résultats et au benchmarking puisque l'on met en concurrence des établissements pour qu'ils atteignent les résultats qu'ils se sont fixés. C'est au moyen de l'évaluation que l'on détermine si ces résultats ont bel et bien été atteints. De plus, dans les deux cas, on place le «client » au centre du système et on lui attribue un rôle capital dans l'évaluation des programmes. L'ensemble de ces caractéristiques laisse à penser que les systèmes de santé du Québec et du Royaume-Uni ne s'inscrivent plus dans ce que Roemer a défini comme le système nationalisé.

\section{Conclusion}

Les réformes que nous avons analysées dans cet article induisent une conception entrepreneuriale des systèmes de santé et révèlent ainsi un changement de paradigme au sein des systèmes nationalisés. Dans le but de réduire les coûts, les deux gouvernements ont imposé les mesures inspirées du nouveau management public que sont la gestion par 
résultats, la prestation de services de qualité, la reddition de compte centré sur le client, l'évaluation en fonction de la satisfaction du client ainsi que le recours aux partenariats publics-privés (PPP). La caractéristique du système nationalisé qui a semblé poser le plus de contraintes est certainement celle de l'unicité de gestion puisque l'abondant recours aux PPP et la recherche de financement provenant du secteur privé ont largement contribué à la transformation des systèmes. Ces changements ont pris des formes différentes dans les deux pays, soit la création de PPP qui ont ouvert la porte à la gestion des hôpitaux britanniques par le secteur privé et, plus récemment au Québec, la création des cliniques affiliées, version québécoise des PPP dans le secteur de la santé. À la lumière de cette analyse, il est dorénavant difficile, surtout depuis l'introduction de l'assurance privée par le gouvernement Charest, mais également depuis l'adoption du nouveau mode de financement du système britannique, de se référer à la typologie de Roemer. En effet, le système nationalisé d'inspiration beveridgienne qui met l'accent sur l'universalisme ainsi que sur l'aspect public du financement et de la prestation des soins ne s'applique plus, selon nous, aux systèmes de santé du Royaume-Uni et du Québec. Il serait plus juste de discuter d'un nouveau modèle de système nationalisé qui emprunte de plus en plus de caractéristiques au système entrepreneurial. Parce que les deux systèmes ont mis en œuvre les éléments du NMP, peut-être pouvons-nous proposer l'existence d'un nouveau modèle : le système managérial. Pour valider la pertinence d'un tel idéal type, il faudra toutefois, aller plus avant en étudiant d'autres systèmes nationalisés comme celui de la Suède.

On peut également se demander si l'ouverture à l'assurance privée au Québec se développera davantage, ce qui rapprocherait le système de santé québécois du système entrepreneurial. En outre, on peut également envisager que, par un processus de social learning (Saint-Martin et Dobrowolsky, 2005), le Royaume-Uni permettra le recours à l'assurance privée; et vice versa, le Québec s'inspirera-t-il peut-être davantage des réformes du Royaume-Uni en poussant un peu plus loin l'application du NMP. Dans la mesure où tous les pays occidentaux a réformé leur système de santé, cette possibilité semble bien réelle.

Mélanie Bourque est professeure au département des sciences administratives de l'UQO.

\section{Bibliographie}

Béland, D, 2005, «Ideas, Interests, and Institutions: Historical Institutionalism Revisited »dans André Lecours (dir.), New Institutionalism: Theory and Analysis, University of Toronto Press, Toronto, pp. 29-50.

Charbonneau J., 2001, «Compte rendu de la Commission d'étude sur les services de santé et les services sociaux. 2000. Les solutions émergentes. Rapport et recommandations », Lien social et politiques, no 46.

Chevallier J., 2003, L'État post-moderne. Librairie générale de droit et de jurisprudence, coll. Droit et société, n³5, 225 p. 
Ferrera M. et A. Hemerjick, 2003 « Recalibrating Europe's Welfare Regimes », dans J. Zeitlin et D. Trubeck (dir.), Governing Work and Welfare in a New Economy, Oxford University Press, Oxford, pp. 88-128.

Hall, P., 1993, «Policy paradigm, social learning and the state : The Case of Economic Policy-Making in Britain », Comparative Politics, vol 25, no. 2, pp. 275-296.

Jenson J., 2003, Converging, Diverging or Shifting? Social Architecture in a Era of Change, the Canadian Political Science Association, Halifax, mai.

Jenson, J., 2006, « Social Investment for New Social Risks: Consequences of the LEGO TM Paradigm for Children», dans Jane Lewis (dir), Children in Context: Changing Families and Welfare States, Edward Elgar Publishing, Toronto.

Lecours, A., 2005, New Institutionalism. Canadian Contributions, University of Toronto Press, Toronto.

Milton J. et M.J. Roemer, 1993, National Health Systems of the World: Issues, vol. 2, Oxford University Press, New York, 356 p.

Mishra, R., 1999, Globalisation and the Welfare State, Edward Elgar, Cheltenham.

Pierson P., 2000, «The Tree World of Welfare Research», Comparative Political Studies, aout-septembre, vol. 33, no. 6-7, pp. 791-821.

Prémont M-C., 2006, « L'affaire Chaoulli et le système de santé du Québec. Cherchez l'erreur. Cherchez la raison », Revue de droit de McGill/McGill Law Journal, vol. 51, pp. 167-195

Rouillard C., É. Montpetit, I. Fortier et A.G. Gagnon, 2004, La réingénierie de l'État : vers un appauvrissement de la gouvernance québécoise, Collection l'espace public, Presses de l'Université Laval, Québec, 200 p.

Quesnel-Vallée A., M. Bourque, C. Fedick et A. Maioni, 2006, « In the aftermath of Chaoulli v. Quebec : Whose opinion prevailed?», Canadian Medical Association Journal, vol. 175, no. 9, octobre.

Rapport de la commission d'enquête sur les services de santé et les services sociaux, Gouvernement du Québec, 1988, Les Publications du Québec, pp. 688-698.

Rapport de la commission d'enquête sur les services de santé et les services sociaux, Gouvernement du Québec, 2001, Les Publications du Québec.

Rivett, J.C., 1998, From Cradle to Grave: fifty years of the NHS, King's Fund, London. 
Saint-Martin D. et A. Dobrowolsky, 2005, « Social Learning, Third Way Politics, and Welfare State Redesign", dans A. Lecours (dir.), New Institutionalism. Canadian Contributions, University of Toronto Press, Toronto. 